\title{
Mark Pieth
}

\section{Braucht Deutschland ein Unternehmensstrafrecht?}

\section{Was hat sich verändert}

Bis vor kurzem schien die Welt der deutschen Dogmatik noch in Ordnung. Kaum jemand zweifelte hier an der Geltung des Grundsatzes „societas delinquere non potest " ${ }^{1}$ Natürlich gab es schon geraume Zeit eine Verbandshaftung, gestützt auf das OWiG. Allerdings wurde der Unterschied zur strafrechtlichen Unternehmenshaftung in der rechtswissenschaftlichen Diskussion intensiv gepflegt. ${ }^{2}$

Im Gefolge der beschleunigten Globalisierung seit den 1990er Jahren wurden immer mehr internationale Instrumente verabschiedet, die eine Unternehmenshaftung voraussetzten (zunächst in den wirtschaftsstrafrechtlichen Themen Geldwäsche, Betrug gegen die Interessen der Europäischen Union, Terrorfinanzierung, organisiertes Verbrechen, Korruption, Cyber-Crime sowie zum Umweltstrafrecht; später wurden weitere Bereiche einbezogen wie Menschenhandel, sexuelle Ausbeutung von Kindern und auch Rassismus). ${ }^{3}$

Die Forderung nach einer Verbandshaftung beruht vor allem auf zwei Überlegungen: Unternehmen sind darauf angewiesen, Aufgaben und damit auch Verantwortung zu delegieren. Mit dieser (erlaubten) Delegation geht allerdings das Risiko einher, dass individuelle Verantwortliche für Fehlverläufe von außen her nicht mehr auszumachen sind. Das ist eine Konsequenz der Komplexität von Unternehmen und muss durchaus nicht das eigentliche Ziel der Delegation sein

1 Böse, Corporate Criminal Liability in Germany, in: Pieth/Ivory (eds.), Corporate Criminal Liability, Emergence, Convergence, and Risk, 2011, 227 (228).

2 Zuletzt vgl. eingehend Kempf/Lüderssen/Volk (Hrsg.), Unternehmensstrafrecht, 2012.

3 Geldwäscherei und Terrorismusfinanzierung: The FATF Recommendations, February 2012, Rec 3-8; Vorschlag für eine Richtlinie des Europäischen Parlaments und des Rates zur Verhinderung der Nutzung des Finanzsystems zum Zwecke der Geldwäsche und der Terrorismusfinanzierung (COM[2013] 45 final vom 5.2.2013), Art. 57 Abs. 4 und 5; Konvention des Europarates über Geldwäsche, Terrorismusfinanzierung sowie Ermittlung, Beschlagnahme und Einziehung von Erträgen aus Straftaten (SEV-Nr. 198 vom 16.5.2005); Organisiertes Verbrechen: UN Convention on Transnational Organized Crime (A/RES/55/25, 15 November 2000); Betrug: Zweites Protokoll aufgrund von Artikel K.3 des Vertrags über die Europäische Union zum Übereinkommen über den Schutz der finanziellen Interessen der Europäischen Gemeinschaften (ABl. C 221 vom 19.7.1997), Art. 3; Bestechung: OECD Convention on Combatting Bribery of Foreign Public Officials in International Business Transactions (adopted 21 November 1997, signed 17 December 1997), Art. 2; UN General Assembly Resolution (A/RES/58/4, 31 October 2003), Annex; United Nations Convention against Corruption, Art. 26; Europarat Strafrechtsübereinkommen über Korruption (SEV-No. 173 vom 27.1.1999), Art. 18; Zweites Protokoll aufgrund von Artikel K.3 des Vertrags über die Europäische Union zum Übereinkommen über den Schutz der finanziellen Interessen der Europäischen Gemeinschaften (ABl. C 221 vom 19.7.1997), Art. 3; Menschenhandel: Richtlinie 2011/36/ EU des Europäischen Parlaments und des Rates vom 5. April 2011 zur Verhütung und Bekämpfung des Menschenhandels und zum Schutz seiner Opfer sowie zur Ersetzung des Rahmenbeschlusses 2002/629/ JI des Rates (ABl. L 101/1 vom 15.4.2011), Art. 5 und 6; Sexuelle Ausbeutung von Kindern: Richtlinie 2011/93/EU des Europäischen Parlaments und des Rates vom 13. Dezember 2011 zur Bekämpfung des sexuellen Missbrauchs und der sexuellen Ausbeutung von Kindern sowie der Kinderpornografie sowie zur Ersetzung des Rahmenbeschlusses 2004/68/JI des Rates (ABl. L 335/1 vom 17.12.2011), Art. 12 und 13; Rassismus: Rahmenbeschluss des Rates 2008/913/JI zur strafrechtlichen Bekämpfung bestimmter Formen und Ausdrucksweisen von Rassismus und Fremdenfendlichkeit vom 28. November 2008 (ABl. L 328/55 vom 6.12.2008), Art 5 und 6. 
(wo allerdings die „strukturelle Unverantwortlichkeit" ${ }^{\text {"4 }}$ gerade bezweckt wird, spricht man auch vom Problem der „Festung Unternebmen“). ${ }^{5}$

Sowohl Wirtschafts- wie Umweltkriminalität haben vielfach ganz erhebliche finanzielle Verluste zur Folge. Sie wirken typischerweise transnational und können Langzeitfolgen nach sich ziehen, die durch individuelle strafrechtliche Haftung nicht adäquat aufgearbeitet werden. ${ }^{6}$ Es ist für das Unternehmen zu leicht, sich vom Einzelnen zu trennen und mit der eingeschliffenen Verbandsattitüde weiterzuarbeiten.

Es versteht sich, dass das Unternehmen selbst nicht schuldfähig im engeren Sinne ist. Allerdings entwickeln Unternehmen ein Betriebsklima und ein Zugehörigkeitsgefühl bei den Angestellten, welche das Verhalten der Einzelnen stark bestimmen können. Das Studium des Korruptionsfalles Siemens ist geradezu ein Lehrbuchbeispiel kollektiver Fehlverläufe im Unternehmen. ${ }^{7}$

\section{Internationale Standards}

Die internationalen Instrumente ${ }^{8}$ haben im ersten Anlauf gar nicht so sehr auf die exakte Konstruktionsform der Unternehmenshaftung geachtet. Insbesondere das Anti-Korruptions-Abkommen der OECD, ${ }^{9}$ das als Impulsgeber diente, nutzt das Prinzip der „funktionalen Äquivalenz “. ${ }^{10}$ Jedes Land hat die Wahl, wie es nach Maßgabe seiner rechtlichen Tradition das vereinbarte Ziel erreichen will. Entscheidend ist, dass Haftungsvoraussetzungen und Sanktion insgesamt „effective, proportionate and dissuasive" ausfallen. Bislang hatte Deutschland keine Schwierigkeiten, gegenüber den internationalen Organisationen auf einer parastrafrechtlichen Lösung zu insistieren. Immerhin war aber die Unternehmenshaftung nach OWiG mit einer Abschöpfung illegaler Gewinne verbunden. Einzelne Staaten, wie Italien, Chile oder Russland, haben den deutschen Ansatz kopiert. Interessanterweise haben aber die anderen Staaten der deutschen Rechtstradition (Österreich ${ }^{11}$ und die Schweiz) ${ }^{12}$ den Schritt zum eigentlichen Unternehmensstrafrecht vollzogen. Mit dem Problem, dass Unternehmen nicht im klassischen Sinne schuldfähig sind, hat man sich hier schon deshalb weniger schwer getan, weil ja bekanntlich auch im überkommenen Individualstrafrecht schuldunabhängige Sanktionen ausgesprochen werden, unter dem Titel der Maßnahmen. ${ }^{13}$

Kotzur, Ausbau der wirtschaftsverwaltungsrechtlichen Unternehmenshaftung, in: Kempf/Lüderssen/ Volk (Fn. 2), 379 (386).

5 Nijboer, A plea for a systematic Approach in Developing Criminal Procedural Law Concerning the Investigation, Prosecution, and Adjudication of Corporate Entities, in: Eser/Heine/Huber (eds.), Criminal responsibility of Legal and Collective Entities, 1999, 301 (317).

6 Dies zu bestreiten wirkt eher hilflos: vgl. Stellungnahme des Deutschen Anwaltsvereins (durch den Ausschuss Strafrecht), zum Entwurf eines Gesetzes zur Einführung der strafrechtlichen Verantwortlichkeit von Unternehmen und sonstigen Verbänden des Landes Nordrhein-Westfalen, Berlin, Dezember 2013, $10 \mathrm{f}$.

7 Graeff/Grieger (Hrsg.), Was ist Korruption? Begriffe, Grundlagen und Perspektiven gesellschaftswissenschaftlicher Korruptionsforschung, 2012; Weidenfeld (Hrsg.), Nützliche Aufwendungen? Der Fall Siemens und die Lehren für das Unternehmen, die Industrie und Gesellschaft, 2011.

8 Vgl. Fn. 3.

9 OECD Convention on Combatting Bribery of Foreign Public Officials in International Business Transactions (adopted 21 November 1997, signed 17 December 1997).

10 Pieth, „Funktionale Äquivalenz“: Praktische Rechtsvergleichung und internationale Harmonisierung von Wirtschaftsstrafrecht, Zeitschrift für Schweizerisches Recht (ZSR) 119/2000, $477 \mathrm{ff}$.

11 Verbandsverantwortlichkeitsgesetz (VbVG von 2006).

12 Art. 102 Schweizer StGB.

13 Stratenwerth, Strafrechtliche Unternehmenshaftung?, in: Festschrift Rodolf Schmitt, 1992, $295 \mathrm{ff}$. 
Der eigentliche Grund, weshalb der internationale Druck gegenüber Deutschland ansteigt, ist eher, weil das klassische OWiG in verschiedener Hinsicht dem internationalen Standard nicht mehr entspricht.

Zwar hat man zwei der augenfälligsten Defizite gerade korrigiert. Man hat die wenig überzeugende Sanktionsobergrenze angehoben, und man hat eine Regelung für die Unternehmensnachfolge gefunden. ${ }^{14}$

$\mathrm{Zu}$ Recht aber hatte der DAV ${ }^{15}$ in seiner Stellungnahme von 2012 moniert, dass ein prinzipielles Überdenken der Verbandshaftung angezeigt sei. Die Gründe für die internationale und auch innerdeutsche Kritik sind eine Reihe von Konstruktionsmängeln der Verbandshaftung nach OWiG. Sie werden in seltener Deutlichkeit auch von der Bundesratsvorlage des Landes Nordrhein-Westfalen herausgearbeitet. ${ }^{16}$ Für unsere Zwecke steht dabei die Frage im Vordergrund, ob diese Defizite prinzipiell gegen eine verwaltungsstrafrechtliche Lösung sprechen. In der Begründung zum Gesetzesantrag des Landes Nordrhein-Westfalen wird darauf verwiesen, dass das OWiG den Verband haften lässt, wenn Organe, Gesellschafter, Prokuristen oder Generalbevollmächtigte nach $\$ 30$ Abs. $1 \mathrm{OWiG}$ die Straftat selbst schuldhaft begehen oder wenn eine Leitungsperson gemäß $\$ 9$ OWiG sich eine Aufsichtsverletzung zu Schulden kommen lässt. Zu Recht verweist der Entwurf darauf, dass nach geltendem Recht eine Verbandsstrafe nach OWiG ausgeschlossen ist, wenn die Tat keiner Einzelperson zugerechnet werden kann bzw. wenn kein schuldhaftes Versagen einer Aufsichtsperson nachzuweisen ist. ${ }^{17}$ Nicht nur bereiten damit systemische Feblentwicklungen Probleme, das OWiG verstößt gegen die Anforderungen etwa der OECD an die Verbandshaftung, da sie zu sehr an ein Individualverschulden geknüpft wird. Die OECD verlangt, dass die Verbandshaftung unabhängig davon greift, ob ein Individuum zur Verantwortung gezogen werden kann (oder könnte). ${ }^{18}$

Die aktuelle Ausgestaltung des OWiG greift auf der einen Seite zu kurz, weil sie zu sehr individuelle Schuld als Grundlage der Unternehmenshaftung ins Zentrum rückt, ${ }^{19}$ auf der anderen Seite verpasst das Modell die Chance, eine Ausnahme für seriöse Compliance-Bestrebungen zu schaffen: Grund der Haftung des Unternehmens sollte nicht das Versagen individueller Leitungsfiguren, sondern die ungenügende Organisation des Unternehmens sein. Das Unternehmen steht dafür ein, dass es Delikte wie die Bezugstat nicht verhindert hat. ${ }^{20}$

Ein weiteres Konfliktfeld ist das Prozessrecht. Während für das Individualstrafrecht nach wie vor das Legalitätsprinzip gilt, wird die Nähe zum Verwaltungsrecht beim OWiG durch die Geltung der Opportunitätsmaxime markiert. ${ }^{21}$ Nun ist zutreffend, dass in der Paxis mancher Länder der "Zwilling“ der Unterneh-

Vgl. das Achte Gesetz zur Änderung des Gesetzes gegen Wettbewerbsbeschränkungen vom 26.6.2013 (BGBl. 2013 I 2013, 1738 ff.); krit. dazu aber Gesetzesantrag des Landes Nordrhein-Westfalen, Entwurf eines Gesetzes zur Einführung der strafrechtlichen Verantwortlichkeit von Unternehmen und sonstigen Verbänden des Landes Nordrhein-Westfalen (E-VerbStrG), 42.

15 Stellungnahme des Deutschen Anwaltsvereins (durch den Ausschuss Strafrecht), Berlin, September 2012, 6 .

16 Gesetzesantrag des Landes Nordrhein-Westfalen (Fn. 14), 23 ff.

17 Gesetzesantrag des Landes Nordrhein-Westfalen (Fn. 14), 23 f., 44 ff.

18 Vgl. zum Verhältnis der Verbandshaftung gemäß Art. 2 OECD Konvention zur Individualhaftung: Pieth, Art. 2 - The Responsibility of Legal Persons, in: Pieth/Low/Bonucci (eds.), The OECD Convention on Bribery, 2014, 212 (233).

19 Zum „Junktim“ zwischen Verbandsverfahren und Individualverfahren: Gesetzesantrag des Landes Nordrhein-Westfalen (Fn. 14), 33, 43 f., 68.

20 Vgl. auch Deutscher Anwaltsverein (Fn. 15), 6.

21 Krit. OECD, Bericht Evaluierung Deutschland Phase 3, 25 f.; vgl. auch Gesetzesantrag des Landes Nordrhein-Westfalen (Fn, 14), 5, 13,23, 67f 
menshaftung der „Deal“ ist. Weiter könnte man sich auch für Deutschland ohne

Weiteres vorstellen, dass die bereits faktisch weitgehend bestehende Opportunität auch im ordentlichen Strafprozess zur Norm erhoben wird, ${ }^{22}$ allerdings ist die Diskrepanz zwischen Individual- und Verbandshaftung schwer zu vertreten. Sie führt denn auch dazu, dass von der Verbandshaftung im Vergleich zur Individualhaftung nur spärlich Gebrauch gemacht wird. ${ }^{23}$

Insgesamt ist damit die Frage, wie es mit der Unternehmenshaftung weitergehen soll, noch nicht geklärt. Der internationale Vergleich zeigt denn auch, dass die Frage nach Strafrecht oder Para-Strafrecht eher zweitrangig ist: Fundamental ist die Grundstruktur der Haftungsnorm und vor allem die Frage, wer die Haftung auslöst. ${ }^{24}$

\section{Modellvielfalt und Annäherung}

Ein Blick auf die ausländischen Modelle der Verbandshaftung zeigt, dass gegenwärtig ein Annäherungsprozess im Gang ist.

Die Vorläufer sind - zur Zeit der Industrialisierung (insbesondere des Eisenbahnbaus $)^{25}$ - im angelsächsischen Rechtsraum zu finden. In den USA wurde die zivilrechtliche Kausalhaftung des Prinzipals für Angestellte und Sklaven ins Strafrecht übernommen (unter dem Begriff der „vicarious liability“). ${ }^{26} \mathrm{Im}$ common law, insbesondere in Grossbritannien, ${ }^{27}$ wurde demgegenüber das Unternehmen für die Handlungen (bzw. Unterlassungen) des Prinzipals selbst haftbar gemacht. Das führte im ersten Anlauf zu einer beschränkten Haftung für Fehlverhalten des obersten Unternehmenschefs ${ }^{28}$ ("identification theory“).

Die kontinentaleuropäischen Modelle, die viel später entwickelt worden sind, nehmen bei beiden Ansätzen Anleihen auf: Wenn der Europarat ${ }^{29}$ sowohl bei der Haftung für kriminelles Verhalten des Organs selbst wie für Verstöße gegen Aufsichtspflichten ansetzt, wird die traditionelle Identifikationstheorie mit Elementen der amerikanischen Haftungsform angereichert. Ein ähnlicher Ansatz findet sich auch im OWiG sowie im neuen Gesetzesantrag des Landes Nordrhein-Westfalen.

In den Voraussetzungen der Haftung wird vorweg die Annäherung der Systeme erkennbar. Zwar bestehen international Differenzen, was die Erfassung der verschiedenen Verbände betrifft (während einzelne Staaten die Rechtsform ins Zentrum rücken, gehen andere von einem wirtschaftlichen Unternehmensbegriff aus). Auch was die Bezugstaten anbelangt, bestehen Unterschiede. Gewisse Rechtsordnungen unterscheiden allenfalls Vorsatztaten und sogenannte „regulatory offences ", 30 andere konzentrieren die Anwendung auf Katalogtaten, überwiegend aus dem Bereich der Wirtschaftsdelikte, ${ }^{31}$ wiederum andere erfassen alle

Strafprozessrecht, Grundriss für Studium und Praxis, 2. Aufl., 2012, $38 \mathrm{ff}$

23 Krit. etwa OECD, Bericht Evaluierung (Fn. 21), , 9, 21 ff. $(25,27)$.

24 Pieth, Ein Europäisches Unternehmensstrafrecht, in: Kempf/Lüderssen/Volk (Fn. 2), 395 (397 f.).

25 Pieth/Ivory (Fn. 1), 4.

26 Coffee, Corporate Criminal Liability: An Introduction and Comparative Survey, in: Eser/Heine/Huber (eds.), Criminal responsibility of Legal and Collective Entities, 1999, 9 (14ff.); Nanda, Corporate Criminal Liability in the United States: Is a New Approach Warranted?, in: Pieth/Ivory (Fn. 1), $63 \mathrm{ff}$. Wells, Corporations and Criminal Responsibility, $2^{\text {nd }}$ edn, 2001.

Vgl. Tesco v. Natrass [1972] AC 153 at 1(Reid LJ).

Vgl. bereits R (88) 18: "Liability of Enterprises having Legal Personality for Offences Committed in the Exercise of their Activities” vom 20. Oktober 1988; aber auch die „Criminal Law Convention on Corruption“, in Kraft seit 1. Juli 2002, Art. 18.

Dazu Pieth/Ivory (Fn. 1), 23.

So etwa Art. 102 Abs. 2 des Schweizer StGB. 
Taten ab einer bestimmten Schwere. Praktisch überall bemüht man sich, Taten ohne Bezug zum Unternehmenszweck oder zu betriebstypischen Risiken auszunehmen.

Die wohl wichtigste Unterscheidung ist die danach, ob jeder Angestellte und Agent des Unternehmens mit seinem Fehlverhalten Haftung auslösen kann (so insb. nach dem Modell der „vicarious liability“) oder ob das Fehlverhalten des Unternehmenschefs, sei es durch die eigene Tat oder durch mangelhafte Aufsicht, die Haftung auslöst (das erweiterte Identifikationsmodell). ${ }^{32}$

Entscheidend ist allerdings, dass immer mehr Staaten dazu übergehen, einen Haftungsnachlass für gute Unternehmensführung vorzusehen. Im Einzelnen kann das auf unterschiedlichem Wege erfolgen:

- Die USA geben für "Sound Corporate Governance“ resp. "Compliance“ einen Strafzumessungsbonus, der in extremis $100 \%$ ausmachen, das heißt zu Straflosigkeit führen kann. ${ }^{33}$

- Der UK Bribery Act 2010 hat in s. 7 einen Rechtfertigungsgrund eingeführt („adequate systems defence“), der allerdings vom Unternehmen dargetan werden muss. ${ }^{34}$

- Die Schweiz geht noch einen Schritt weiter und verlangt vom Staatsanwalt den Nachweis der Desorganisation als eigentliche Haftungsvoraussetzung. ${ }^{35}$

Insgesamt setzt sich weltweit ein sogenannter due diligence approach to corporate liability ${ }^{36}$ durch.

\section{Nachbolbedarf für Deutschland?}

Mit Blick auf diese Entwicklung drängt sich die Frage nach einem allfälligen Nachholbedarf im deutschen Recht auf.

Das hauptsächliche Defizit der aktuellen deutschen Lösung liegt in der Konstruktion des OWiG begründet: Eine Verbandshaftung, die zwar auf eine Bezugstat abstellt, die allerdings das Unternehmen dafür verantwortlich macht, dass es ein riskantes Betriebsklima toleriert oder das Fehlverhalten gar gefördert hat, würde nicht auf verschuldetes Fehlverhalten von Leitungsfiguren abstellen, sondern - wesentlich objektiver - die Desorganisation an sich thematisieren: Hat das Unternehmen es unterlassen, alle zumutbaren Vorkehrungen zu treffen, um Delikte, die dem typischen Betriebsrisiko entsprechen, zu verhindern?

Vgl. OECD Recommendation for further Combatting Foreign Bribery of Foreign Public Officials in International Business and Administration Transactions, adopted by the Council on 26 November 2009, Annex I B).

33 US Sentencing Guidelines (USSG): United States Sentencing Commission 2012, Revised Guidelines Manual, 1 November 2012; FCPA Resource Guide 2012, A Resource Guide to the U.S. Foreign Corrupt Practices Act by the Criminal Division of the U.S. Department of Justice and the Enforcement Division of the U.S. Securities and Exchange Commission, 14 November 2012; dazu Pieth (Fn. 18), $239 \mathrm{f}$.

34 O'Shea, The Bribery Act 2010, A Practical Guide, 2011, $129 \mathrm{ff}$.; Raphael, The Bribery Act 2010, $2010,57 \mathrm{ff}$.

35 Zum Schweizerischen Art. 102 Abs. 2 StGB vgl. Jean-Richard-dit-Bressel, Das Organisationsdelikt. Art. 102 Abs. 2 StGB im internationalen Kontext, 2013; Forster, Die strafrechtliche Verantwortlichkeit des Unternehmens nach Art. 102 StGB, 2006; Geiger, Organisationsmängel als Anknüpfungspunkt im Unternehmensstrafrecht: aufgezeigt am Beispiel Geldwäschereibekämpfung im Private Banking einer Bank-AG, 2006; Heine, Das kommende Unternehmensstrafrecht (Art. 100quater f.), ZStrR 1/2003, 24 ff.; Pieth, Internationale Anstöße zur Einführung einer strafrechtlichen Unternehmenshaftung in der Schweiz, ZStrR 119/2001, 1 ff.; ders., Die strafrechtliche Verantwortung des Unternehmens, ZStrR 121/2003, 353 ff.; ders., Risikomanagement und Strafrecht, Organisationsversagen als Voraussetzung der Unternehmenshaftung, in: Sutter-Somm/Hafner/Schmid/Seelmann (Hrsg.), Risiko und Recht. Festgabe zum Schweizerischen Juristentag 2004, 2004, 597 ff.; ders., Strafverfahren gegen das Unternehmen, in: Festschrift Albin Eser, 2005, $599 \mathrm{ff}$. 
Anders als die UK Bribery Act 2010 hat auch der Gesetzesentwurf von Nordrhein-Westfalen nicht den Mut, sich auf Unternehmensebene von der Fiktion einer Verbandsschuld zu befreien. ${ }^{37}$ Entgegen $\$ 2$ (2) des Entwurfs eines Verbandsstrafgesetzbuchs (E-VerbStrG) ist der Bezug auf „vorsätzliches oder fahrlässiges Aufsichts- oder Überwachungsverschulden “38 unnötig, da $\$ 5$ einen Exzeptionsbeweis für „ausreichende organisatorische und personelle Maßnahmen“ vorsieht: Eine doppelte Freizeichnung, einmal, weil generell ein seriöses Compliancesystem bestand und der Anlasstäter ein sogenannter "rogue employee“ war, und zusätzlich noch, weil die Leitungsfigur kein eigenes persönliches Verschulden trifft, ist übermäßig. Damit fällt der E-VerbStrG, entgegen der eigenen Kritik, in die Falle des geltenden Rechts.

Die Briten hatten dies im Laufe der Ausarbeitung der UK Bribery Act 2010 rechtzeitig gemerkt und an die Stelle einer bloß erweiterten „identification theo$r y^{{ }^{* 39} 9}$ in section 7 eine Kausalhaftung mit eigentlicher Rechtfertigungsmöglichkeit vorgesehen. ${ }^{40}$

\section{Nochmals: Strafrecht oder Alternativen?}

Die Frage, die die Gemüter hierzulande nach wie vor am meisten zu beschäftigen scheint ${ }^{4}{ }^{4}$ soll aber nicht einfach unbeantwortet bleiben. Internationalrechtlich besteht gegenwärtig (noch) keine Verpflichtung zur Einführung eines Unternehmensstrafrechts, ${ }^{42}$ eine Verbandssanktion im Sinne des OWiG, wenn auch eines grundsätzlich überarbeiteten $\mathrm{OWiG}$, könnte auch längerfristig international gesehen ausreichen. Immerhin wird der politische Druck auch deshalb zunehmen, weil die Nachbarn Schwierigkeiten bei der Rechtshilfe in Strafsachen erwarten.

Allerdings ist eine grundsätzliche Unmöglichkeit, in Deutschland eine strafrechtliche Unternehmenshaftung einzuführen, nicht erkennbar, sonst müsste man auch mit dem strafrechtlichen Maßnahmenrecht auf unüberwindbare verfassungsrechtliche Probleme stoßen.

Jetzt fragt sich, welche Lösung zweckmäßiger ist. Die Erfahrung der europäischen Staaten, die die strafrechtliche Unternehmenshaftung kennen, ist vielfach, ${ }^{43}$ dass die Praxis Hemmungen hat, bei Unternehmen zum Strafrecht zu greifen, während die Erfahrung in Deutschland mit dem OWiG eher in die Gegenrichtung zeigt: Gerade weil nicht eine strafrechtliche Sanktion ausgesprochen wird, fällt es leichter, sogar sehr hohe Geldsanktionen, bisweilen bis an den Rand der Existenzvernichtung auszusprechen. Auch prozessual macht man es sich mit dem OWiG wesentlich einfacher. Echtes Strafrecht würde volle Verteidigungsrechte bedingen.

Indessen besteht eine schwer erklärbare Diskrepanz zwischen unserer lockeren Hand, mit der wir Alltags- und Elendskriminalität - wohlgemerkt im Individualbereich - mit Strafrecht abhandeln und unserem enormen rechtsstaatlichen

\footnotetext{
Vgl. auch Deutscher Anwaltsverein (Fn. 6), $16 \mathrm{f}$.

So aber der Gesetzesantrag des Landes Nordrhein-Westfalen (Fn. 14), 8, 45 f.

Law Commission, Reforming Bribery (Law Com No 313), $97 \mathrm{ff}$.

Oben Fn. 34.

41 BDI/BDA, Stellungnahme zum Entwurf eines Gesetzes zur Einführung der strafrechtlichen Verantwortlichkeit von Unternehmen und sonstigen Verbänden vom 31. Januar 2014; Deutscher Anwaltsverein (Fn. 6), $15 \mathrm{ff}$.

42 Pieth (Fn. 24), 397.

43 Sowohl in Österreich wie in der Schweiz ist die Verbandsstrafe bisher sehr zurückhaltend angewandt worden. Die Praxis hat Anlass zu kriminologischen Untersuchungen gegeben, die allerdings noch im Gange sind.
} 
Aufwand, mit dem wir die Möglichkeit einer Unternehmensstrafe erörtern. ${ }^{44}$ Dabei bestreitet kaum jemand, dass typischerweise das Risikopotential für die Gesellschaft bei Unternehmensstraftaten (seien es Wirtschafts- oder Umweltdelikte) ein Vielfaches der klassischen Individualstraftaten ausmacht.

Gelegentlich wird vorgeschlagen, dem Dilemma dadurch zu entgehen, dass mit Unternehmen zwar streng zu verfahren sei, man aber zur „moralisch neutralen“ Abschöpfungssanktion im Sinne des Kartellrechtes greift. ${ }^{45}$ Dabei ist gerade die ethisch-moralische Neutralität suspekt. Der Schritt, Delikte unternehmensstrafrechtlich zu erfassen, wollte in den angelsächsischen Staaten durchaus als Appell verstanden werden, es sollte der Ruf des Unternehmens in Frage gestellt werden. Interessanterweise nehmen Unternehmenschefs das auch heute noch so wahr. In der neuesten Ausgabe des „PwC 2014 Global Economic Crime Survey"46 befürchteten $36 \%$ der befragten Unternehmenschefs schwere Rufschäden bei strafrechtlicher Verurteilung wegen Bestechung, während - bei vergleichbarer Sanktionshöhe - bei Kartellvergehen lediglich 21 \% um den Ruf ihres Unternehmens fürchteten. Es ist davon auszugehen, dass sowohl Manager wie das weitere $\mathrm{Pu}-$ blikum die neutrale Gewinnabschöpfung wesentlich weniger ernst nehmen als die Unternehmensstrafe im eigentlichen Sinne.

Um ein letztes Mal die Parallele zur Maßnahme im Individualstrafrecht zu bemühen: Wir hätten auch dort die Wahl, bei fehlender Schuld ganz ins präventive Verwaltungsrecht auszuweichen - in extremis eine verwaltungsrechtliche Verwahrung anzuordnen. Das tun wir bewusst nicht, weil der Ausgangspunkt der Begehung einer Straftat uns wichtig scheint: Mit der strafrechtlichen Maßnahme sollen die Voraussetzungen nach den klaren Maßstäben des Strafprozessrechtes festgestellt werden, und es soll ein Unwerturteil ${ }^{47}$ über die Tat an sich durchaus aufrechterhalten werden, auch wenn mangels Schuld Strafe im technischen Sinne nicht ausgesprochen werden kann oder - je nach Rechtsordnung - aufgeschoben werden muss.

Allerdings ist damit die inzwischen klassisch gewordene Fragestellung nach Strafrecht versus Aliud ${ }^{48}$ nicht abschließend beantwortet. Wenn die Haftungsgrundlage dem traditionellen Strafrecht nachempfunden sein sollte, heißt das nicht, dass auch das gesamte Sanktionsarsenal strafrechtlich sein muss: Im Sinne eines holistischen Ansatzes ${ }^{49}$ sollte neben finanziellen Sanktionen (Geldstrafe, Einziehung, Verfall) mehr Raum für Sanktionsformen geschaffen werden, die weniger Drittschäden produzieren und vor allem die Zukunft des Unternehmens weniger beeinträchtigen. Im Vordergrund steht der „Compliance Monitor“"50 des angelsächsischen Rechts, der als Bewährungshelfer einen Wandel der kriminogenen Betriebsattitüde befördern kann. ${ }^{51}$

Vgl. nochmals BDI/BDA (Fn. 41), Deutscher Anwaltsverein (Fn. 6), 32; in der Literatur nur Kempf/ Lüderssen/Volk (Fn. 2).

45 Lüderssen, Interventionsrecht gegen Unternehmen? - Skizze eines neuen Haftungstypus, in: Kempf/ Lüderssen/Volk (Fn. 2), $387 \mathrm{ff}$.

46 www.pwc.com/crimesurvey.

47 Zum Gesichtspunkt des „Unwerturteils“ vgl. auch den Gesetzesantrag des Landes Nordrhein-Westfalen (Fn. 14), 25.

48 Hassemer, Kennzeichen und Krisen des modernen Strafrechts, ZRP 1992, 378 (383); ders., Person, Welt und Verantwortlichkeit. Prolegomena einer Lehre von der Zurechnung im Strafrecht, in: Lüderssen (Hrsg.), Aufgeklärte Kriminalpolitik oder Kampf gegen das Böse?, 1998, 350 (368).

49 Pieth, Co-Regulierung im Wirtschaftsstrafrecht, in: Festschrift Winfried Hassemer, 2010, 891 ff. (896).

50 Zur Literatur: Pieth, in: Pieth/Low/Bonucci (Fn. 18), Nanda (Fn. 26), $80 \mathrm{ff}$.

51 Vgl. auch den Gesetzesantrag des Landes.Nordrhein-Xestfalen (Fn. 14), 59 f. 